studies end each chapter and serve well to illustrate the more theoretical emphasis of preceding sections.

This is a good book - comprehensive and replete with practical strategies to hone what one already does as a CBT therapist, but refreshingly with more of an eye on outcome rather than process, which can rather overwhelm some of the more manualised approaches. The authors deal with evidence in an even-handed manner, and it is this balance coupled with a wealth of practical tips that contribute to this book's strong clinical utility and likely longevity.

Durham, R. C., Chambers, J. A., Power, K. G., et al (2005) Long-term outcome of cognitive behaviour therapy (CBT) clinical trials in central Scotland. Health Technology Assessment, 9, I-174.

James B. Woolley PO67, Institute of Psychiatry, De Crespigny Park, Denmark Hill, London SE5 8AF, UK. Email: j.woolley@iop.kcl.ac.uk

doi: 10.1192/bjp.189.5.474a

\section{Sleep Psychiatry}

Edited by A. Golbin, H. Kravitz \& L. Keith. London: Taylor and Francis. 2004. 412 pp. EII0.00 (hb). ISBN I842I4I457

Sleep provides the foundation for daytime alertness, attention and learning. It is not a passive state but a complex, active cyclical process controlled by elegant mechanisms. The emerging specialty of sleep medicine utilises the expertise of respiratory physicians, neurologists, psychiatrists and psychologists. Sleep architecture may be disrupted in confusional states and affective, anxiety and neurodevelopmental disorders. With the recent revision of the
International Classification of Sleep Disorders (ICSD), there is a clear need to incorporate research literature into a readily accessible text for practising clinicians. The introduction of this hardback book exploring the relationship between sleep and psychiatry is both timely and necessary.

There are 28 well-referenced chapters written by an international panel of experts. The text is structured to examine sleep physiology with particular attention to ontogenesis, REM (rapid eye movement) sleep and gender differences (section 1); developmental, psychiatric and forensic aspects of sleep (sections 2-5); and a concluding section offering a review of pharmacological and 'alternative' treatments.

The text provides a good review of insomnia and an interesting theoretical discussion exploring the relationship between REM sleep and search activity, providing a conceptual framework for anxiety and affective disorders. Sleep in developmental disorders is comprehensively reviewed although the clinical implications of reported sleep abnormalities often remain unclear. Perhaps the authors should be applauded for including a review of 'Normal and abnormal dreams' as this remains a subject of much controversy and ongoing curiosity. However, I felt the content was weighted to psychodynamic explanations and the evidence to suggest reliably identifiable characteristics of 'abnormal' dreams lacked rigour. An adaptive theory of parasomnias using chaos theory as a template argues that 'chaos' in cerebral and other (e.g. cardiac) regulatory centres is advantageous, allowing the flexibility necessary to perform regulatory functions. Parasomnias are considered as chaotic behaviours that reinstate cerebral control to produce a normal sleep pattern. The concept is further developed to incorporate the relationship between daytime habits, arousal and dissociation, although alternative explanations merit due consideration. A useful discussion of forensic sleep psychiatry is also provided.

Unfortunately, there is no overview of the ICSD, which would help to provide a contextual framework for the psychiatric aspects of sleep disorders. The text would also benefit from a chapter dedicated to the clinical and sleep laboratory assessment and investigation of sleep disorders. Much of the relevant information is dispersed in the various chapters but this is not an entirely user-friendly approach. Valiant efforts are made to overcome the lack of definitive research data. At times, however, the text transgresses into unnecessary detail, or even self-indulgence, without providing a sufficiently coherent overview, and thus lacks clarity. The expression of subjective views, which are acknowledged to be based upon personal clinical experience, does require a more robust analysis of the evidence, such as the stipulation that unusual childhood sleep positions may be predictive of later somatic problems. Repetition can become a little tiresome. It is not clear whether this is a deliberate strategy of reinforcement or an inevitable consequence of multiple contributing authors.

Although the text was a little disappointing, perhaps trying too hard and in so doing, losing focus at times, it remains worthwhile reading for any psychiatrist with an interest in sleep abnormalities. However, practising clinicians may find that access to information relating to the clinical management of sleep disorders requires some effort to filter out less relevant information.

Manny Bagary Consultant Neuropsychiatrist, Queen Elizabeth Psychiatric Hospital, Mindelsohn Way, Edgbaston, Birmingham BI5 2QZ, UK. Email: manny.bagary@bsmht.nhs.uk

doi: 10.I192/bjp.189.5.475 